\title{
Bio-maleimide-stained plasma microparticles can be purified in a native state and target human proximal tubular $\mathrm{HK}_{2}$ cells
}

\author{
MOHAMMAD J. ALKHATATBEH ${ }^{1}$, LISA F. LINCZ ${ }^{2}$ and RICK F. THORNE ${ }^{3}$ \\ ${ }^{1}$ Department of Clinical Pharmacy, Faculty of Pharmacy, Jordan University of Science and Technology, Irbid 22110, Jordan; \\ ${ }^{2}$ Hunter Haematology Research Group, Calvary Mater Newcastle Hospital, Waratah, NSW 2298; \\ ${ }^{3}$ School of Environmental and Life Sciences, University of Newcastle, Ourimbah, NSW 2258, Australia
}

Received September 14, 2016; Accepted October 20, 2016

DOI: $10.3892 /$ br.2016.806

\begin{abstract}
Plasma microparticles (MPs) are heterogeneously sized submicron extracellular vesicles that originate from the cell membrane as a result of cell activation or apoptosis. Circulating MPs express cell-specific molecules that reflect their cell of origin and they are increasingly investigated for their potential role in intercellular communication. The aim of the current study was to determine if size exclusion chromatography could be used to purify fluorescent-labeled MPs in sufficient concentrations to be used experimentally in cell binding assays. Bio-maleimide was used to stain plasma MPs in platelet free plasma before applying to size exclusion chromatography. Collected fractions were analyzed for protein content and MPs were enumerated by flow cytometry. Fractions were ultracentrifuged and MPs further confirmed by western blotting for the putative diabetic marker, cluster of differentiation (CD)36 and platelet-specific CD41 proteins. Fractions that contained MPs were incubated with $\mathrm{HK}_{2}$ cells to determine MP-cell binding. Bio-maleimide-stained MPs were detected across various fractions of size exclusion, and pellets of these fractions confirmed positivity for the MP markers, CD41 and CD36. The addition of the isolated MPs to $\mathrm{HK}_{2}$ renal tubular cells and analysis by epi-fluorescent imaging demonstrated that, in principle, the labeled MPs are able to bind to cells in vitro. Notably, only the first eluted MP fraction bound $\mathrm{HK}_{2}$ cells indicating a possible association between MP size and cell-targeting properties.
\end{abstract}

\section{Introduction}

Plasma microparticles (MPs) are small extracellular vesicles that originate from the membrane of cells undergoing activation or apoptosis (1). In addition to phosphatidylserine (PS),

Correspondence to: Dr Mohammad J. Alkhatatbeh, Department of Clinical Pharmacy, Faculty of Pharmacy, Jordan University of Science and Technology, Irbid 22110, Jordan

E-mail:khatatbeh@just.edu.jo

Key words: bio-maleimide, microparticles, size, targeting a phospholipid membrane component that is exposed on the surface of MPs of different cell origins, circulating MPs exhibit cell-specific proteins that reflect their parent cells (2). For example, cluster of differentiation (CD)41-, CD14-, CD235a- and CD31-expressing MPs are considered to be platelet, monocyte, erythrocyte and endothelial cell-derived MPs, respectively (3). Additionally, plasma MPs have been found to carry other parental molecules, including nucleic acids and lipids (2). As revealed by Arraud et al (4), plasma MPs detected by electron microscopy were reported to be heterogeneous in size and the majority of them were detected to be in the size range, $0.03-0.9 \mu \mathrm{m}$ in diameter.

Plasma is most abundant in platelet MPs, which were initially described in 1967 as 'platelet dust', representing the sediment of plasma ultracentrifugation (5). The function of these platelet-derived particles was not completely understood, however, they were believed to possess pro-coagulant properties (5). Subsequently, MPs were identified to be involved in various biological processes, including inflammation, blood coagulation and cell signaling; functions, which are mediated by their unique structure and expression of certain cell-specific molecules (6). Furthermore, MPs are overproduced in various pathological conditions where inflammation and vascular dysfunction are implicated, indicating that MPs may serve as surrogate biomarkers for these diseases (6). Among these, endothelial MPs are usually elevated in diseases that are associated with endothelial dysfunction, including diabetes mellitus and atherosclerosis $(7,8)$. Additionally, increased generation of specific subgroups of plasma MPs may be associated with certain pathological conditions, such as CD36-positive MPs, which were found to be elevated in patients with type 2 diabetes mellitus (9).

Notably, increasing evidence indicates that plasma MPs are involved in intercellular communication (10). Plasma MPs may act as vehicles of parental proteins, nucleic acids, lipids and other molecules, which are delivered to recipient cells where they may influence various physiological and pathological processes (11). For example, MPs may initiate certain signaling pathways in the target cell by direct ligand-surface receptor binding or by being internalized into the target cell (2). The exact mechanism by which MPs target other cells has not been sufficiently determined; however, it is proposed to be mediated by ligand-receptor binding (12). This 
was investigated in previous research using endothelial MPs generated in vitro to target endothelial cells (12) or other cell types, such as the human proximal tubular $\mathrm{HK}_{2}$ cells (13).

A lack of methodology for isolating specific MP subsets from plasma has resulted in limited research in this area. Specifically, to the best of our knowledge, there have been no previous studies investigating the significance of MP size on its cell binding. In addition, these heterogeneously sized MPs pose many technical challenges in their analysis and functional evaluation. Flow cytometry is a mainstay for MP analysis, however the application of flow cytometric sorting is limited as a method to analyze the whole MP population, which is heterogeneous in size. Rather, for bulk MP preparation, ultracentrifugation is the mainstay approach $(12,13)$. However, the coalescence of membranous material in a pellet requires considerable mechanical disruption to resuspend and there are questions as to whether this is a true MP suspension. In the current study, the aim was to use size exclusion chromatography to isolate plasma MPs (14) as an alternative technique, which purifies MPs in a native state and uses these MPs in cell binding assays. An additional aim was to refine this technique to allow further fractionation of MPs according to their size and to test the ability of these different sized MPs to bind to cells in vitro. The human proximal tubular $\mathrm{HK}_{2}$ cell line was used, as it was successfully targeted by endothelial MPs, as described by Fernandez-Martinez et al (13) and because renal tubular cells are involved in the development of diabetic nephropathy. Furthermore, the present study hypothesized that the diabetic marker, CD36, may contribute to the pathophysiology of the disease by mediating MP binding to cellular targets. Thus, MP expression of CD36 was investigated in the present study, as well as the more abundant platelet marker, CD41, when characterizing different sized MP fractions.

\section{Materials and methods}

Antibodies, immunoblotting and other reagents. Bio-maleimide (Bodipy ${ }^{\circledR}$ FL N-(2-aminoethyl) maleimide) was obtained from Thermo Fisher Scientific, Inc. (Waltham, MA, USA). Mouse monoclonal antibody against CD36 (prepared by Dr Rick Thorne, as previously described (15); clone long 9; used for western blotting) and the immunoblotting method (enhanced chemiluminescence) were performed as previously described (15). Briefly, a homemade enhanced chemiluminescence solution $(100 \mathrm{mM}$ Tris hydrochloride (pH 8.8), $0.4 \mathrm{mM}$ p-Coumaric acid, $2.5 \mathrm{mM}$ luminol and $2.6 \mathrm{mM} \mathrm{H}_{2} \mathrm{O}_{2}$; Sigma-Aldrich, Castle Hill, Australia) was used and the chemiluminescence reaction was detected using an LAS-4000 imaging system (Fuji Photo Film Co., Tokyo, Japan). The polyclonal antibody against platelet CD41 was a gift from Dr Pidard (Unite de Pharmacologie Cellulaire, Unite Associee IP/INSERM 285, Institut Pasteur, Paris).

Blood sampling and plasma processing. Fresh whole blood samples (5 ml each) were collected from healthy volunteers by venipuncture with 21-gauge needles and transferred into centrifuge tubes containing EDTA as the anticoagulant at a final concentration of $2 \mathrm{mM}$. Blood samples were immediately double centrifuged at $3,000 \mathrm{x}$ g for $15 \mathrm{~min}$ at room temperature to prepare the platelet free plasma (PFP). The upper-third of the PFP supernatant was carefully collected by pipetting, and filtered using a $1.2-\mu \mathrm{m}$ filter to remove any platelet contamination.

Preparation of bio-maleimide-labeled plasma MPs. Bio-maleimide was used to stain MPs in PFP as previously described (16) with certain modifications. Briefly, a working solution of bio-maleimide was prepared by adding $1 \mu \mathrm{l}$ of $5 \mathrm{mM}$ bio-maleimide stock solution in dimethyl sulfoxide to $49 \mu \mathrm{l}$ phosphate-buffered saline (PBS; 1:50). Thereafter, the diluted bio-maleimide solution was added to $1 \mathrm{ml}$ PFP sample and incubated for $15 \mathrm{~min}$ in the dark at room temperature.

Isolation of bio-maleimide stained plasma MPs by size exclusion. Bio-maleimide-stained PFP (1 ml) was applied to a Sephacryl S-500 HR size exclusion column (40x0.7 cm; GE Healthcare Australia Pty., Ltd., Parramatta, Australia) connected to a BioLogic liquid chromatography system (BioRad Laboratories PTY Ltd., Gladesville, Australia). PBS (pH 7.4) was used as a mobile phase at a flow rate of $0.5 \mathrm{ml} / \mathrm{min}$. Twenty-one elution fractions of $1 \mathrm{ml}$ each were collected and kept in the dark for further experiments. The protein concentrations of all fractions were measured using a BCA Protein Assay Reagent kit (Thermo Fisher Scientific, Scoresby, Australia; lower limit of detection, $20 \mu \mathrm{g} / \mathrm{ml}$ ). The void volume fractions (200 $\mu \mathrm{l}$ of each; 1-11) were ultracentrifuged for $1 \mathrm{~h}$ at $4^{\circ} \mathrm{C}$ at $100,000 \mathrm{x} \mathrm{g}$ in a TLA 100 rotor (Beckman Coulter Australia Pty Ltd., Gladesville, Australia) to isolate MPs as pellets.

Flow cytometric analysis of MPs. MP analysis was performed as previously described (17) and according to the guidelines that were established by the International Society on Thrombosis and Haemostasis, Inc. Vascular Biology Scientific and Standardization Committee on the Standardization of platelet-derived MP enumeration by flow cytometry (18) with modifications suggested for the BD FACS Canto (BD Biosciences, San Jose, CA, USA), including calibration to set MP detection limits (9). To detect Bio-maleimide-stained MPs, 10- $\mu$ l aliquots of bio-maleimide-treated PFP size exclusion fractions were diluted in PBS that did not contain $\mathrm{Ca}^{+2}$ or $\mathrm{Mg}^{+2}$ and were analyzed by flow cytometry. Absolute MP numbers were determined using TruCount counting tubes (BD Biosciences) and events were collected for $60 \mathrm{sec}$ at a low flow rate as previously described (9).

Cell culture and MP-cell binding assay. The human kidney proximal tubular epithelial cell line $\left(\mathrm{HK}_{2}\right)$ was obtained from Dr. Carol Pollock (Department of Medicine, Royal North Shore Hospital, St. Leonards, Australia). This $\mathrm{HK}_{2}$ cell line was originally obtained from American Type Culture Collection (Manassas, VA, USA). Cells were allowed to attach to $13-\mathrm{mm}$ circular glass coverslips in 24-well cell-culture plates and maintained in Dulbecco's modified Eagle's medium (Sigma-Aldrich) that contained $2 \mathrm{mM}$ L-glutamine (Lonza Australia Pty., Ltd., Mount Waverley, Australia) and 4.5 g/l glucose (Sigma-Aldrich) and supplied with $10 \%$ fetal bovine serum (FBS; Sigma-Aldrich), $0.1 \%$ penicillin/streptomycin (Invitrogen; Thermo Fisher Scientific, Inc.), 25 mM HEPES (4-(2-hydroxyethyl)-1-piperazineethanesulfonic acid) and 


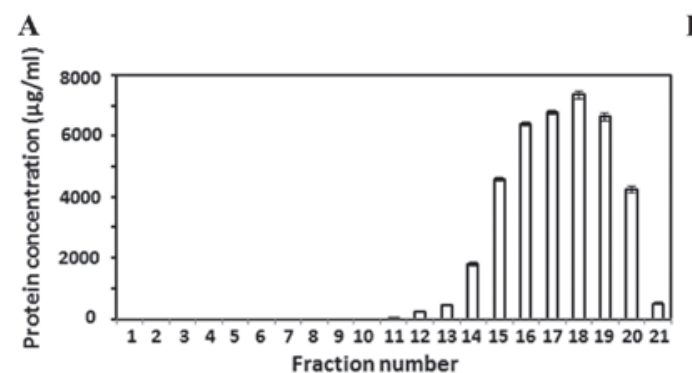

B

C
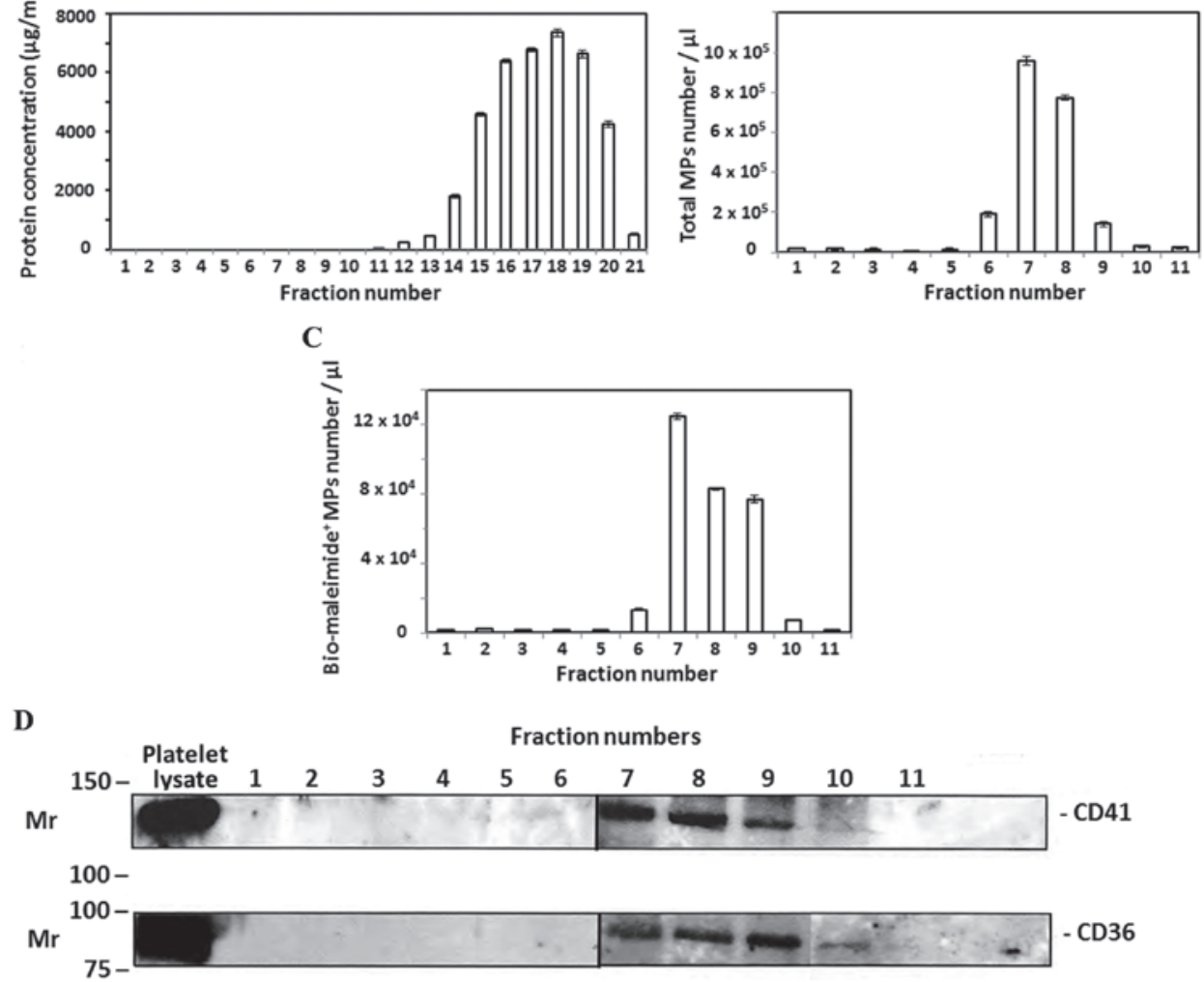

Figure 1. Detection of plasma MPs isolated by size exclusion chromatography. (A) Protein determination of plasma size exclusion fractions showed that the first eleven fractions contain almost no detectable protein. (B) Bar graphs of the total numbers of MPs in the first 11 fractions of plasma size exclusion. (C) Bar graphs show numbers of bio-maleimide-stained MPs in the first 11 fractions of plasma size exclusion. (D) Plasma size exclusion fractions were ultracentrifuged to isolate suspended MPs. Pellets were analyzed by western blotting for CD36 and the platelet-specific CD41. CD36 and CD41 were detected in fractions that contained MPs whereas other fractions were negative for the two proteins. Bars represent the means \pm standard deviation of three experiments. MP, microparticle; CD, cluster of differentiation.

$24 \mathrm{mM}$ sodium hydrogen carbonate (Lonza Australia Pty., Ltd.). Cells were incubated at $37^{\circ} \mathrm{C}$ in a humidified atmosphere of $5 \% \mathrm{CO}_{2}$ in air (v/v). At $\sim 50 \%$ confluence, cells were deprived of FBS for 2-3 $\mathrm{h}$ and incubated with $0.5 \mathrm{ml}$ of each of the bio-maleimide-stained size exclusion fractions for a further hour. Cells were washed twice using PBS to remove unbound MPs and were fixed with $4 \%$ formaldehyde for $5 \mathrm{~min}$. Thereafter, cells were washed twice using PBS before staining with 4',6-diamidino-2-phenylindole (DAPI; Sigma-Aldrich) (19). The coverslips were mounted on microscope slides and cells were imaged under a fluorescence microscope.

\section{Results}

Isolation of bio-maleimide stained plasma MPs by size exclusion chromatography. Size exclusion chromatography is an effective method for isolating MPs from other plasma components without ultracentrifugation (14). As plasma MPs are large in size (0.1-1 $\mu \mathrm{m}$ in diameter), MPs are expected to be eluted from the size exclusion column before the plasma proteins (14). In the current study, bio-maleimide-stained PFP were fractionated by size exclusion chromatography into 21 fractions and the protein concentration in each fraction was determined. As shown in Fig. 1A, plasma proteins were detected in fractions 12-21 without any protein detected in the first 11 fractions. By contrast, flow cytometric analysis of the same fractions detected the majority plasma MPs in fractions 7-9 (Fig. 1B) without any MP detection in fractions 12-21 (data not shown). In addition, plasma MPs were successfully stained with bio-maleimide as a general MP stain as previously described (16) and enumerated in PFP size exclusion fractions 1-11 by flow cytometry (Fig. 1C). Plasma MPs carry molecules that are specific to their parent cells, such as cell-surface receptors and proteins (2). PFP size exclusion fractions 1-11 were ultracentrifuged to isolate the suspended MPs. Western blot analysis was performed for CD36 and the platelet-specific protein, CD41. As shown in Fig. 1D, MP pellets of size exclusion fractions 7-9 were positive for CD36 and CD41. This indicates that MPs isolated by size exclusion chromatography may originate from platelets, as they were expressing CD41, and from other cells, as CD36 is not only expressed by platelets, but also by other cell types, such as red blood cells (9). However, detecting plasma MPs in various size exclusion fractions indicates that MPs in these fractions were different sizes and bio-maleimide was able to stain the MPs regardless of their size.

Plasma MPs isolated by size exclusion chromatography target $\mathrm{HK}_{2}$ cells in vitro. To further investigate the function of plasma MPs isolated by size exclusion chromatography, size exclusion fractions that contained bio-maleimide-stained MPs (Fig. 1) were 
A
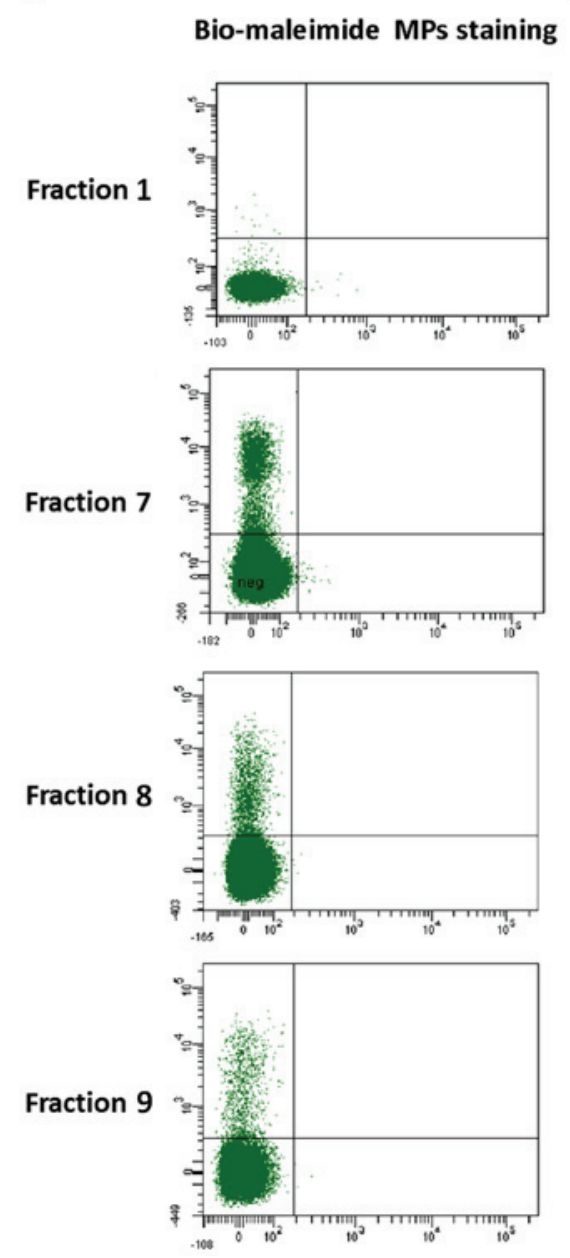

B
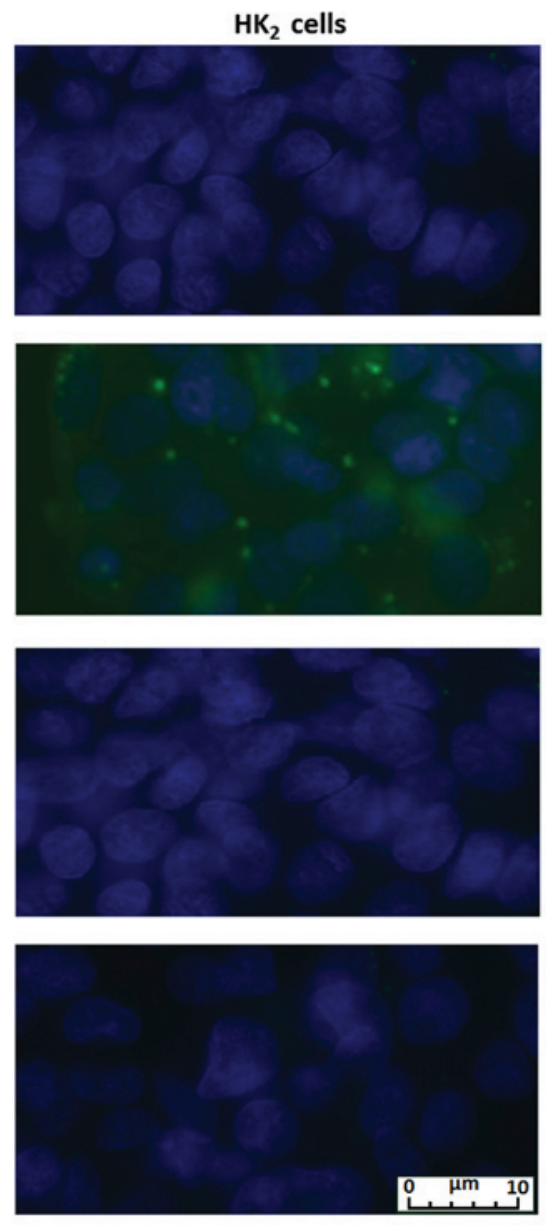

Figure 2. Plasma microparticles (MPs) isolated by size exclusion target $\mathrm{HK}_{2}$ cells in vitro. (A) Flow-cytometric analysis of plasma MPs isolated from a healthy donor. Plasma was stained using Bodipy ${ }^{\circledR}$ FL N-(2-aminoethyl) maleimide and fractionated by size exclusion chromatography. To detect MPs, fractions were analyzed by flow cytometry, demonstrating MPs enriched in the void volume (fractions 6-10), but absent in the other fractions (Fig. 1). The first representation (fraction 1) is shown as the control, followed by fraction numbers 7, 8 and 9 . Upper left quadrant, MPs stained with bio-maleimide; lower left quadrant, $>90 \%$ of MP events, which may be considered as noise (i.e., not true MPs, as they did not show any MP staining). (B) Fluorescence microscopic images of HK 2 cells treated with the control (fraction 1), void volume fraction 7 stained with bio-maleimide (green), fraction numbers 8 and 9 (these were bio-maleimide-stained, but did not show any MP binding to $\mathrm{HK}_{2}$ cells). As highlighted, the small green dots are consistent with the size of the MPs $(<1 \mu \mathrm{m})$ and are attached to the $\mathrm{HK}_{2}$ cells treated with void volume fraction 7. The cell nuclei were counterstained with 4',6-diamidino-2-phenylindole (blue).

incubated with $\mathrm{HK}_{2}$ cells in vitro. MPs were previously reported to act as vehicles that transport molecules from the cell of origin to other target cells (11). As shown by fluorescence microscopic images of $\mathrm{HK}_{2}$ cells treated with the control, size exclusion fraction 1, and fraction 7 (Fig. 2); the isolated bio-maleimide-stained MPs in fraction 7 were able to attach to $\mathrm{HK}_{2}$ cells. By contrast, $\mathrm{HK}_{2}$ cells treated with fractions number 8 and 9, that also contained bio-maleimide-stained MPs, did not show any fluorescence indicative of MP attachment (Fig. 2). As the concentrations of bio-maleimide-stained MPs in fractions 7 and 8 were $\sim 60-70 \%$ of the MPs concentration of fraction 7, which contained the maximum MP concentration (Fig. 1C), this indicates that MP attachment to $\mathrm{HK}_{2}$ cells was most likely to be dependent on MP size rather than MP concentration.

\section{Discussion}

The current study demonstrates that size exclusion chromatography using a Sephacryl S-500 HR column is a simple technique to isolate circulating MPs from other plasma constituents. Similarly, other studies have shown that size exclusion chromatography using different matrices, including Sepharose CL-2B (20), Sepharose CL-4B and Sephacryl S-400 (21), was able to purify plasma MPs. This indicates that MP isolation by size exclusion chromatography may be performed using different matrices as long as the size range of MPs is larger than the matrix pore size range, which was originally designed to separate protein molecules rather than larger components, such as MPs. Thus, plasma MPs are expected to be eluted from the column in the void volume without crossing the pores of size exclusion matrices (22). However, the aim from using size exclusion chromatography in the present study was to fractionate plasma MPs into various fractions, which are supposed to contain MPs of different sizes. According to the theory of size exclusion chromatography, larger plasma MPs are expected to be eluted from the column prior to smaller sized MPs. As a result, the technique used in the present study was able to fractionate plasma MPs into three 
major fractions (7-9) in which the MP content was confirmed by flow cytometric analysis (Fig. 1C) and western blotting of proteins CD41 and CD36 in the MP pellet of size exclusion fractions following ultracentrifugation (Fig. 1D). Consistently, Böing et al (22) demonstrated that platelet-derived vesicles were successfully isolated in early consecutive fractions of Sepharose CL-2B size exclusion chromatography, as confirmed by flow cytometric analysis and western blotting of proteins specific to platelet-derived vesicles. However, the column dimensions in the current study support better MP isolation according to size, as it was longer and narrower than that used in the study by Böing et al (22).

In addition, results from the current study have confirmed the efficacy of using bio-maleimide as a general stain for plasma MP detection and quantification by flow cytometry (16). Bio-maleimide attaches to thiol groups and cysteine residues of the MPs' membranes and it has a fluorescent nature that can be detected by flow cytometry (13). Notably, bio-maleimide-treated PFP was fractionated, to the best of our knowledge, for the first time by size exclusion chromatography to isolate bio-maleimide-stained plasma MPs from other plasma components. The purpose of bio-maleimide staining was to detect MPs by flow cytometry, rather than using Annexin V staining as an alternative method (16) and to enable fluorescent detection of MPs when they were investigated for in vitro cell targeting.

Although plasma MPs were previously reported to act as vehicles for intercellular communication (10), factors that affect cell targeting were not exactly determined. In the current study, the effect of plasma MP size on $\mathrm{HK}_{2}$ cell targeting was investigated. Unexpectedly, results demonstrated that plasma MPs isolated in different size exclusion fractions possessed different binding abilities to $\mathrm{HK}_{2}$ cells in vitro. MPs isolated in fraction 7, which were presumably larger (as they were eluted from the size exclusion column before other MPs in fractions 8 and 9), were the only MPs that were able to attach to $\mathrm{HK}_{2}$ cells (Fig. 2B). This suggests that MP attachment to $\mathrm{HK}_{2}$ cells may be size dependent or that the MP concentrations in fractions 8 and 9 were insufficient to demonstrate cell binding by fluorescence microscopy. However, as the content of bio-maleimide-stained MPs in fraction 8 and 9 was $>60 \%$ of the content of fraction 7 (Fig. 1C), this supports that MP concentration was less likely to affect MP binding to $\mathrm{HK}_{2}$ cells.

Results from the present study indicate that plasma MPs may be fractionated using size exclusion chromatography and that MPs in these fractions may have different functions. However, there were certain limitations of the current study that may affect the generalization of its results. First, the exact size of plasma MPs was not determinable in each fraction of size exclusion chromatography. It is possible to determine MP sizes by transmission electron microscopy, however, a lack of equipment and funding were the main barriers for this determination. Furthermore, although cell targeting appeared to be independent of MP expression of CD36 and/or CD41, blocking studies are required to confirm this. However, the effect of MP size on cell targeting requires further investigation using multiple cell lines and animal models. In addition, further studies are required to investigate differences between plasma MPs of varying sizes in term of molecular content and surface expression of proteins. This may explain why these MPs may have different functions.

In conclusion, the current study has shown that plasma MPs stained with bio-maleimide may be isolated using Sephacryl S-500 HR size exclusion chromatography; a simple and timesaving technique as compared with ultracentrifugation. Plasma MPs were detected by flow cytometry and western blotting in three consecutive fractions suggesting that these fractions contain MPs of different sizes according to the theory of size exclusion chromatography. Further investigations on the function of these MPs have shown that MPs eluted in the earliest fraction were able to target $\mathrm{HK}_{2}$ cells in vitro. This suggests that MP attachment to $\mathrm{HK}_{2}$ cells may be size-dependent and, thus, different sizes of MPs may have different functions.

\section{Acknowledgements}

The present study was supported by the HMRI Research Grant (grant no. 10-08) funded by the Lions District 201 N3 Diabetes Foundation.

\section{References}

1. Hargett LA and Bauer NN: On the origin of microparticles: From 'platelet dust' to mediators of intercellular communication. Pulm Circ 3: 329-340, 2013.

2. Yáñez-Mó M, Siljander PR, Andreu Z, Zavec AB, Borràs FE, Buzas EI, Buzas K, Casal E, Cappello F, Carvalho J, et al: Biological properties of extracellular vesicles and their physiological functions. J Extracell Vesicles 4: 27066, 2015.

3. Nielsen MH, Beck-Nielsen H, Andersen MN and Handberg A: A flow cytometric method for characterization of circulating cell-derived microparticles in plasma. J Extracell Vesicles 3: Feb 4, 2014 (Epub ahead of print). doi: http://dx.doi. org/10.3402/jev.v3.20795.

4. Arraud N, Linares R, Tan S, Gounou C, Pasquet JM, Mornet S and Brisson AR: Extracellular vesicles from blood plasma: Determination of their morphology, size, phenotype and concentration. J Thromb Haemost 12: 614-627, 2014.

5. Wolf $\mathrm{P}$ : The nature and significance of platelet products in human plasma. Br J Haematol 13: 269-288, 1967.

6. Lynch SF and Ludlam CA: Plasma microparticles and vascular disorders. Br J Haematol 137: 36-48, 2007.

7. Horstman LL, Jy W, Jimenez JJ and Ahn YS: Endothelial microparticles as markers of endothelial dysfunction. Front Biosci 9: 1118-1135, 2004.

8. Bernard S, Loffroy R, Sérusclat A, Boussel L, Bonnefoy E, Thévenon C, Rabilloud M, Revel D, Moulin P and Douek P: Increased levels of endothelial microparticles CD144 (VE-Cadherin) positives in type 2 diabetic patients with coronary noncalcified plaques evaluated by multidetector computed tomography (MDCT). Atherosclerosis 203: 429-435, 2009.

9. Alkhatatbeh MJ, Enjeti AK, Acharya S, Thorne RF and Lincz LF: The origin of circulating CD36 in type 2 diabetes. Nutr Diabetes 3: e59, 2013.

10. Jaiswal R, Raymond Grau GE and Bebawy M: Cellular communication via microparticles: Role in transfer of multidrug resistance in cancer. Future Oncol 10: 655-669, 2014.

11. Antonyak MA and Cerione RA: Microvesicles as mediators of intercellular communication in cancer. Methods Mol Biol 1165: 147-173, 2014.

12. Jansen F, Yang X, Hoyer FF, Paul K, Heiermann N, Becher MU, Abu Hussein N, Kebschull M, Bedorf J, Franklin BS, et al: Endothelial microparticle uptake in target cells is annexin I/phosphatidylserine receptor dependent and prevents apoptosis. Arterioscler Thromb Vasc Biol 32: 1925-1935, 2012.

13. Fernandez-Martínez AB, Torija AV, Carracedo J, Ramirez R and de Lucio-Cazaña FJ: Microparticles released by vascular endothelial cells increase hypoxia inducible factor expression in human proximal tubular HK-2 cells. Int J Biochem Cell Biol 53: 334-342, 2014. 
14. Alkhatatbeh MJ, Mhaidat NM, Enjeti AK, Lincz LF and Thorne RF: The putative diabetic plasma marker, soluble CD36, is non-cleaved, non-soluble and entirely associated with microparticles. J Thromb Haemost 9: 844-851, 2011.

15. Thorne RF, Zhang X, Song C, Jin B and Burns GF: Novel immunoblotting monoclonal antibodies against human and rat CD36/fat used to identify an isoform of CD36 in rat muscle. DNA Cell Biol 25: 302-311, 2006.

16. Enjeti AK, Lincz L and Seldon M: Bio-maleimide as a generic stain for detection and quantitation of microparticles. Int $\mathrm{J}$ Lab Hematol 30: 196-199, 2008.

17. Robert S, Poncelet P, Lacroix R, Arnaud L, Giraudo L, Hauchard A, Sampol J and Dignat-George F: Standardization of platelet-derived microparticle counting using calibrated beads and a Cytomics FC500 routine flow cytometer: A first step towards multicenter studies? J Thromb Haemost 7: 190-197, 2009.

18. Lacroix R, Robert S, Poncelet P, Kasthuri RS, Key NS and Dignat-George F; ISTH SSC Workshop: Standardization of platelet-derived microparticle enumeration by flow cytometry with calibrated beads: Results of the International Society on Thrombosis and Haemostasis SSC Collaborative workshop. J Thromb Haemost 8: 2571-2574, 2010.
19. Sanchez-Niño MD, Fernandez-Fernandez B, Perez-Gomez MV, Poveda J, Sanz AB, Cannata-Ortiz P, Ruiz-Ortega M, Egido J, Selgas R and Ortiz A: Albumin-induced apoptosis of tubular cells is modulated by BASP1. Cell Death Dis 6: e1644, 2015.

20. de Menezes-Neto A, Sáez MJ, Lozano-Ramos I, Segui-Barber J, Martin-Jaular L, Ullate JM, Fernandez-Becerra C, Borrás FE and Del Portillo HA: Size-exclusion chromatography as a stand-alone methodology identifies novel markers in mass spectrometry analyses of plasma-derived vesicles from healthy individuals. J Extracell Vesicles 4: 27378, 2015.

21. Baranyai T, Herczeg K, Onódi Z, Voszka I, Módos K, Marton N, Nagy G, Mäger I, Wood MJ, El Andaloussi S, et al: Isolation of Exosomes from Blood Plasma: Qualitative and Quantitative Comparison of Ultracentrifugation and Size Exclusion Chromatography Methods. PLoS One 10: e0145686, 2015.

22. Böing AN, van der Pol E, Grootemaat AE, Coumans FA, Sturk A and Nieuwland R: Single-step isolation of extracellular vesicles by size-exclusion chromatography. J Extracell Vesicles 3: Sep 8, 2014. http://dx.doi.org/10.3402/jev.v3.23430. 